\title{
Patient reported symptoms of cancer treatment and patient satisfaction in BNGO practices - a German survey including the NCCN distress thermometer
}

\author{
Jörg Schilling, Alexandra Hansen, Petra Ortner, Mascha Pömmerl
}

For the German Professional Association of Gynaeco-Oncology in Practices (BNGO)

\section{Background and objectives}

Providing high-quality treatment for patients with gynaecologic malignancies on an outpatient basis and maintaining patients' quality of life are pivotal objectives of the German Professional Association of Gynaeco-Oncology in Practices (BNGO). Therefore, Gynaeco-oncologists associated in the BNGO perform biannual quality assurance surveys on patient satisfaction with treatment and care and on the burden of disease and cancer treatment.

In 2015, questions about the frequency of adverse effects of cancer treatment and patient reported burden of side effects and symptoms were added to the questionnaire. In 2017, questions of the NCCN distress thermometer ${ }^{2}$ were additionally included. The survey took place in the period September-December 2017.

\section{Methods}

In August 2017, 11,900 printed questionnaires were distributed to 113 practices. Documentation period was 3 months. 1,116 patients treated in 32 practices completed the 8-pagequestionnaire including questions on the distress they were experiencing, on tumor- and treatment-related symptoms, and on their satisfaction with the practice and the physician. Patients used pictograms to assess their satisfaction with practice, physician and medical staff. In order to assess the distress caused by their cancer treatment in the past weeks, patients used the NCCN distress thermometer.

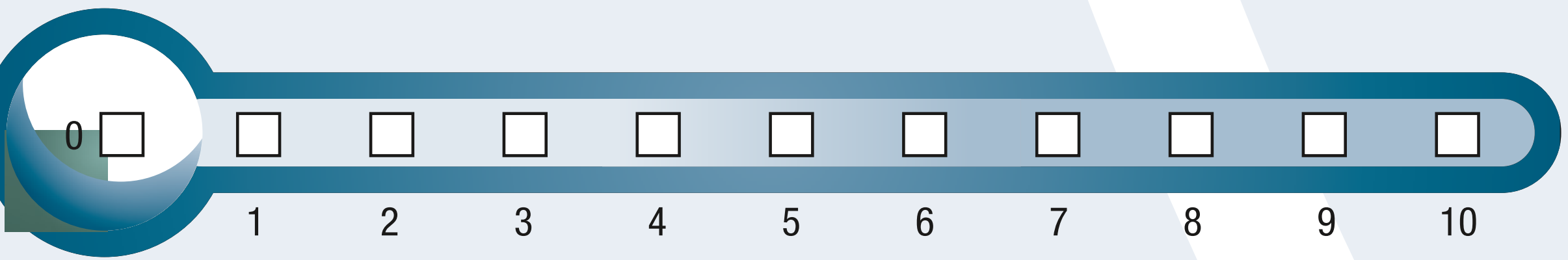

All patients reported the frequency of the symptoms and side effects they experienced using the categories never, seldom, sometimes, often and always/all the time. Patients also reported the burden of the symptoms and side effects using a Visual Analog Scale (VAS) from 1 (least) - 5 (most) or 10 (most) Chemotherapy patients reported the frequency of side effects of chemotherapy and rated the most distressing symptoms on a scale of 1 (least) to 10 (most).

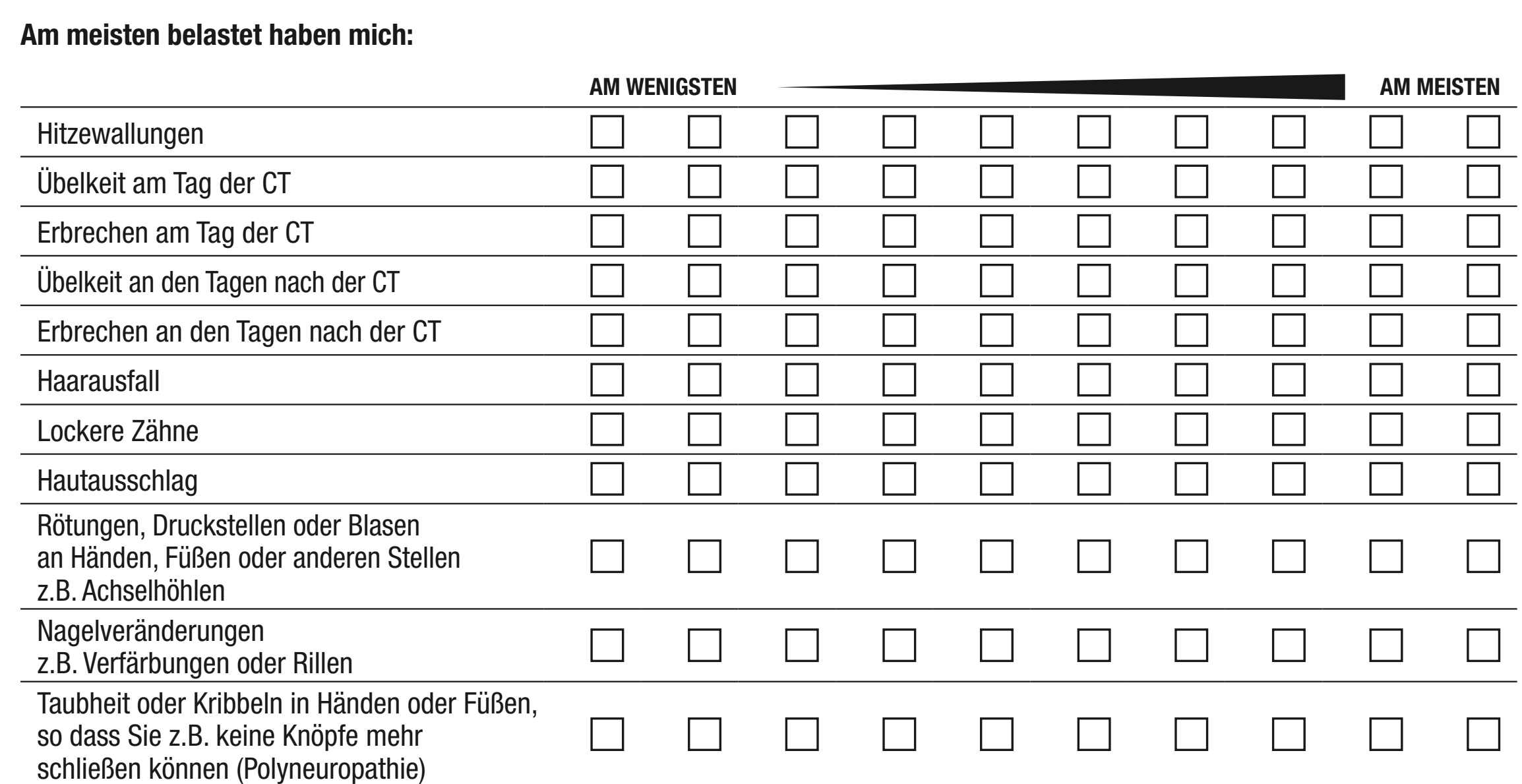

At the end of the questionnaire, patients were asked for a final overall assessment of their cancer treatment and were asked for suggestions for improvements.

\section{Results}

Age and treatment

The majority of patients were aged $50-59$ years $(30.5 \%)$, $25.6 \%$ of the 1,116 patients were aged $60-69$ years.

At the time of the survey, almost $69 \%$ of patients received chemotherapy and $32 \%$ received a drug-based treatment other than chemotherapy. $39 \%$ received radiation therapy and $55 \%$ had undergone surgery.

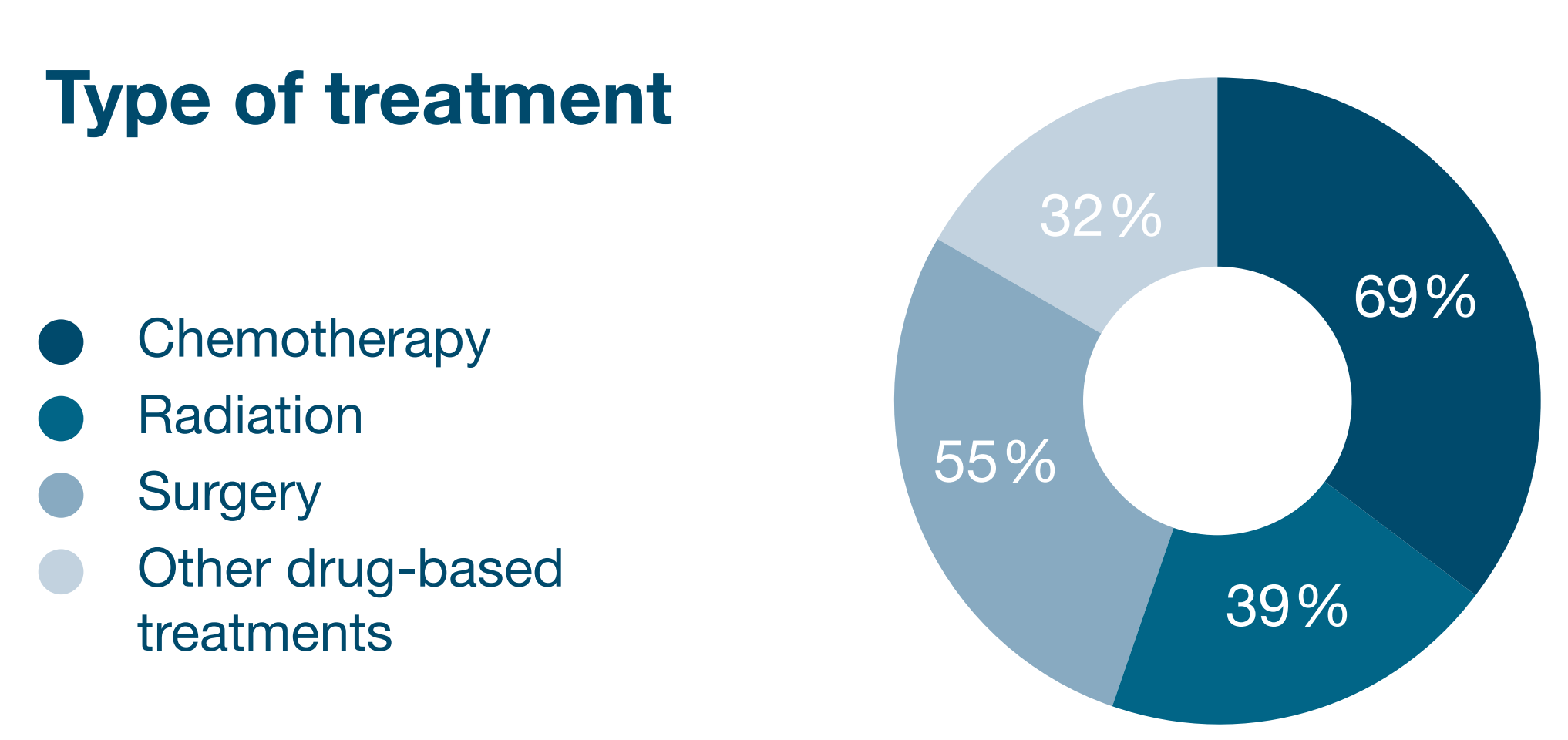

Satisfaction with practice and physician

Equipment and organisation of the practice were assessed as "very good" or "good" by more than $90 \%$ of women and the practice staff and physicians were rated "very good" or "good" by $99 \%$ of patients. $67 \%$ of patients said they would choose outpatient treatment again, $31.7 \%$ did not specify. Of all patients who gave an overall assessment of their cancer treatment, $100 \%$ said they would seek treatment in the same practice again

\section{Overall distress}

How much distress have you been experiencing in the past weeks? $(n=936)$

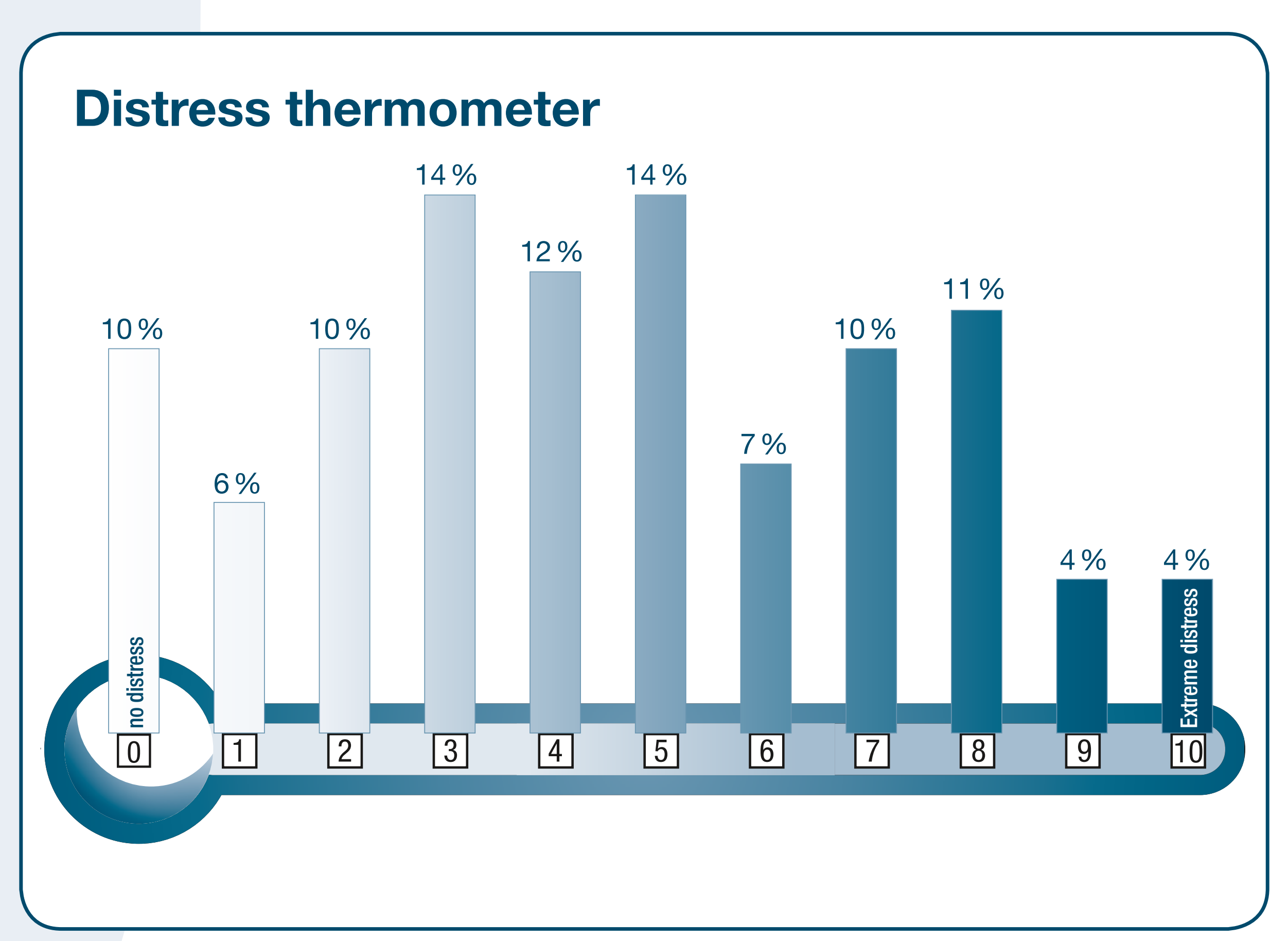

Daily problems, $\mathrm{n}=1000$

In the week prior to the survey, almost half of the patients experienced fears $(45 \%)$, worries (43\%), sadness (34\%), and nervousness (31\%).

The most common physical symptoms included fatigue, problems sleeping, pain and paraesthesia:

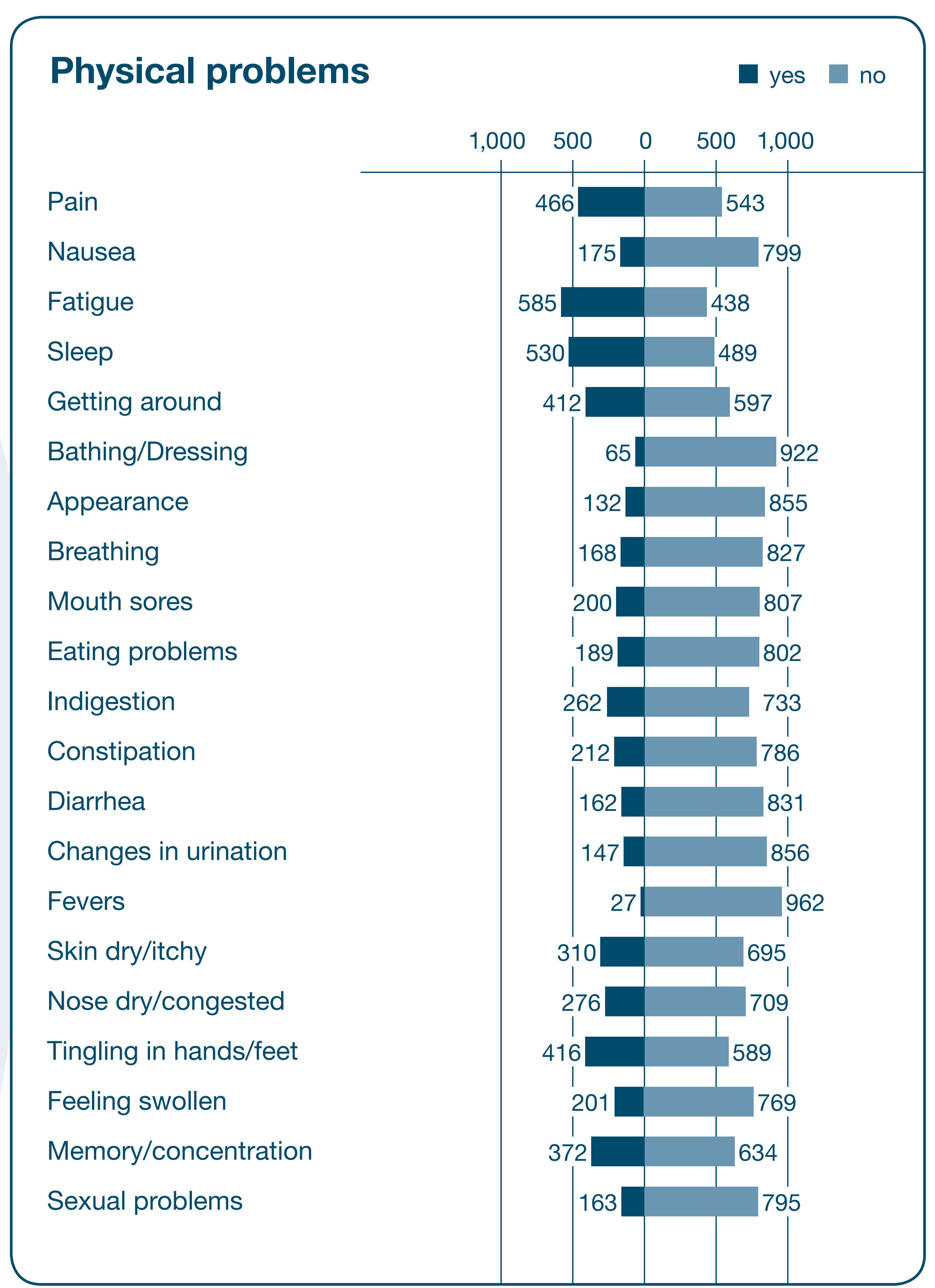

Problems after the end of chemotherapy

Problems persisting after the end of chemotherapy, $n=425$ Always or often

Tiredness and fatigue

Numbness and tingling in hands/feet

Bone pain

Sleeping disorders

ressing disease symptoms, $\mathrm{n}=\mathbf{9 3 8}$ Very or most distressing

Fatigue

blems sleepin

Tredness

Hot flushes

Side effects and symptoms chemotherapy patients

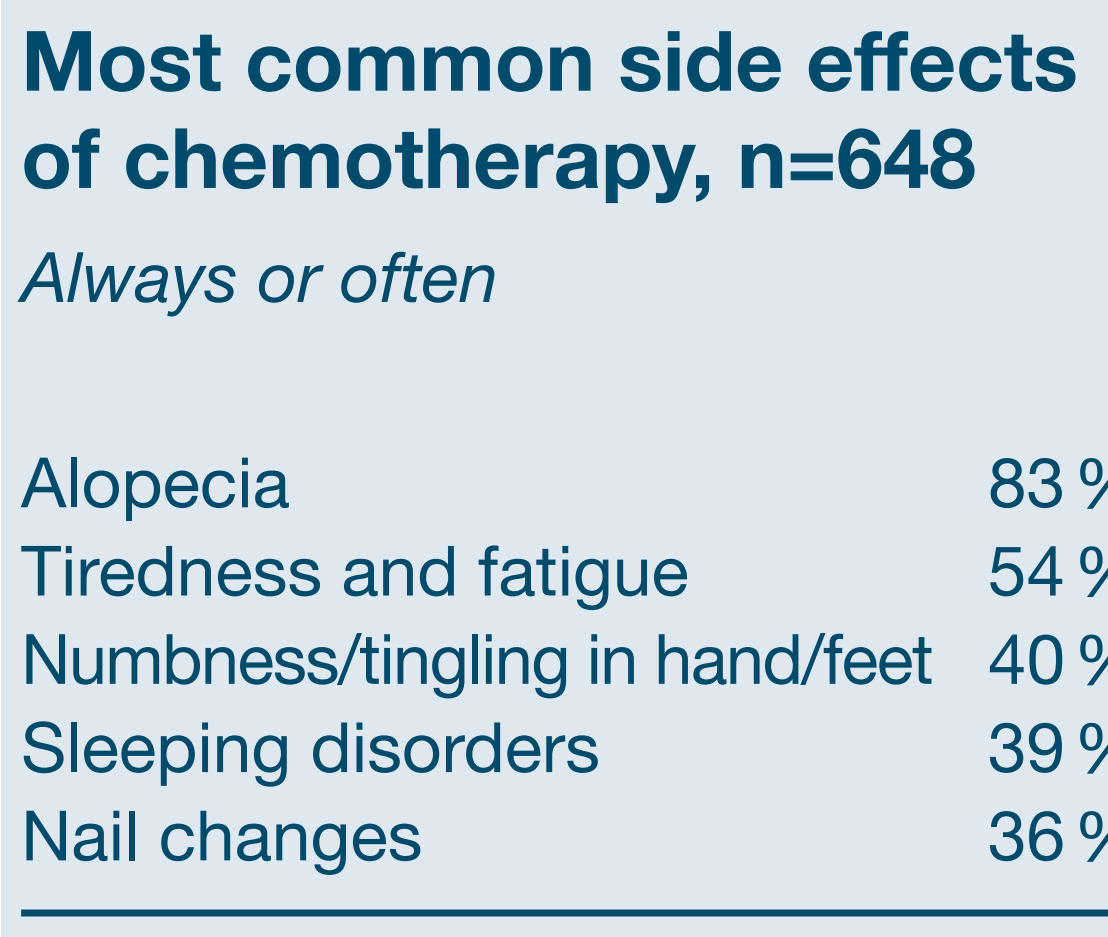

Most distressing side efects of chemotherapy, $\mathrm{n}=626$

Very or most distressing

Bone pain

Numbness/tingling

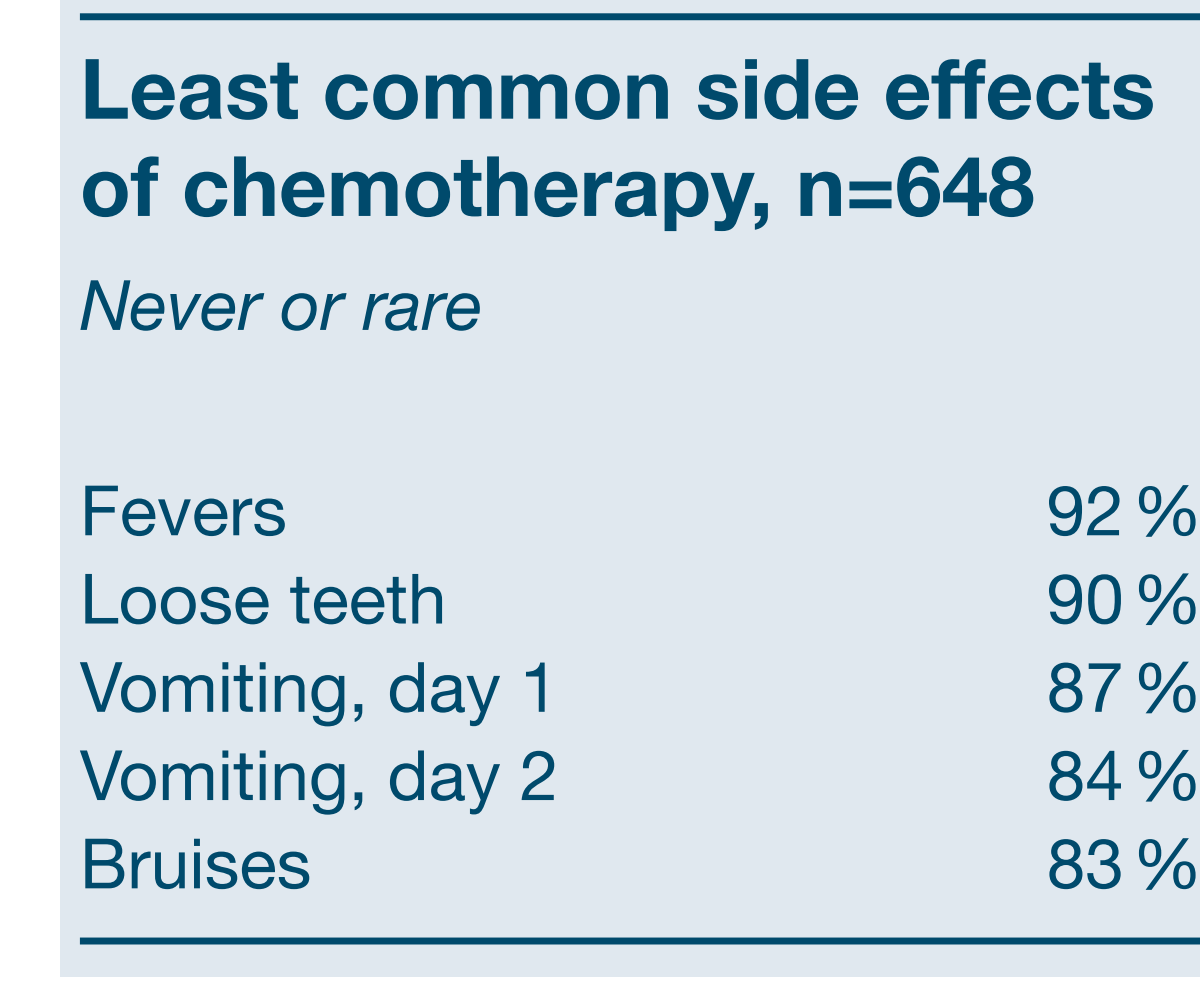

Least distressing side effects of chemotherapy, $\mathrm{n}=626$

Less or least distressing

Fever

Vomiting, day 1

Vomiting, day 1

Bruises

Complementary therapies

$19 \%$ of patients used complementary medication during thei cancer treatment, $37 \%$ of patients did not specify. In $51 \%$ o patients, complementary drugs were recommended by thei oncologist and in $31 \%$ by another physician.

Use of complementary medication

yes

not stated

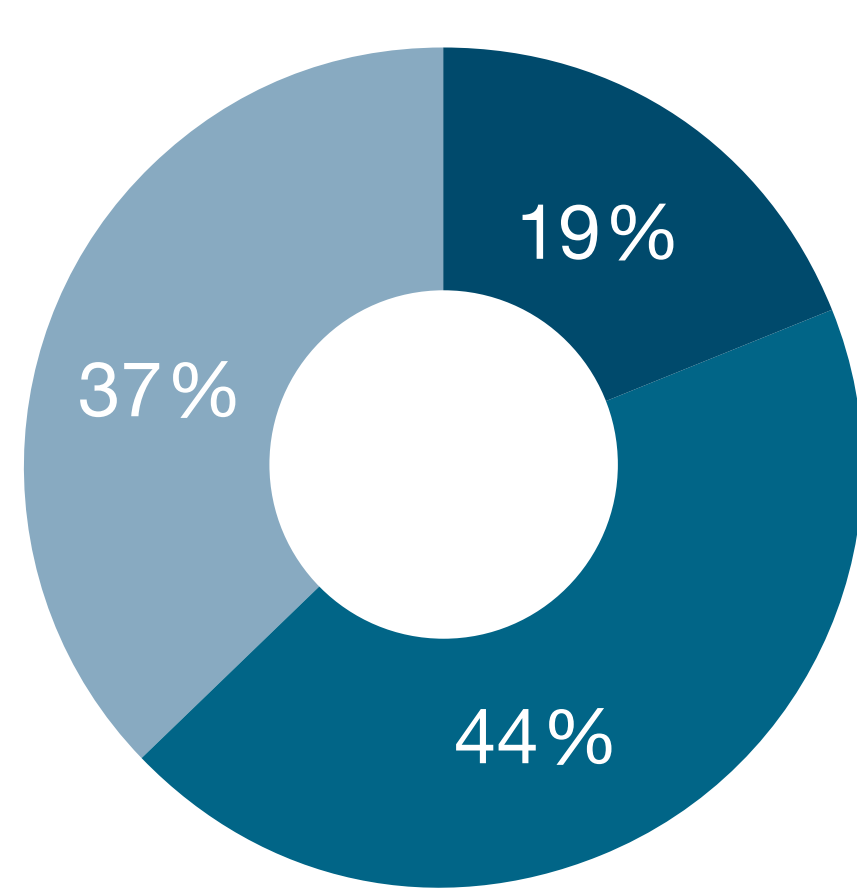

\section{Conclusion}

According to the NCCN distress thermometer included in the survey, $66 \%$ of patients described the distress they experienced due to their cancer or cancer treatment using the categories 0-6 (no distress at all - moment using the categories $0-6$ (no distress at all - mo-
derate distress). Side effects of chemotherapy that can be prevented by supportive care such as nausea and emesis are among the least common side effects. Alopecia is the most common side effect of chemotherapy and is also the second most distressing symptom after bone pain. of patients. $40 \%$ of patients had paraesthesia (always or very often) during chemotherapy that persisted in 34\% of patients even after they had finished chemotherapy. 